Research Article

\title{
Towards Energy-Efficient Framework for IoT Big Data Healthcare Solutions
}

\author{
Chong Feng (iD, ${ }^{1}$ Muhammad Adnan, ${ }^{2}$ Arshad Ahmad ${ }^{1},{ }^{1,3}$ Ayaz Ullah, \\ and Habib Ullah Khan ${ }^{4}$ \\ ${ }^{1}$ School of Computer Science \& Technology, Beijing Institute of Technology, Beijing, China \\ ${ }^{2}$ Department of Computer Science, Abdul Wali Khan University, Mardan, Pakistan \\ ${ }^{3}$ Department of Computer Science, University of Swabi, Anbar, Swabi, Pakistan \\ ${ }^{4}$ Department of Accounting \& Information Systems, Qatar University, Doha, Qatar \\ Correspondence should be addressed to Chong Feng; fengchong@bit.edu.cn
}

Received 22 February 2020; Revised 23 May 2020; Accepted 26 May 2020; Published 12 June 2020

Academic Editor: Iván García-Magariño

Copyright ( $\odot 2020$ Chong Feng et al. This is an open access article distributed under the Creative Commons Attribution License, which permits unrestricted use, distribution, and reproduction in any medium, provided the original work is properly cited.

\begin{abstract}
The aim of the Internet of things (IoT) is to bring every object (wearable sensors, healthcare sensors, cameras, home appliances, smart phones, etc.) online. These different objects generate huge data which consequently lead to the need of requirements of efficient storage and processing. Cloud computing is an emerging technology to overcome this problem. However, there are some applications (healthcare) which need to process data in real time to improve its performance and require low latency and delay. Fog computing is one of the promising solutions which facilitate healthcare domain in terms of reducing the delay multihop data communication, distributing resource demands, and promoting service flexibility. In this study, a fog-based IoT healthcare framework is proposed in order to minimize the energy consumption of the fog nodes. Experimental results reveal that the performance of the proposed framework is efficient in terms of network delay and energy usage. Furthermore, the authors discussed and suggested important services of big data infrastructure which need to be present in fog devices for the analytics of healthcare big data.
\end{abstract}

\section{Introduction}

Nowadays the Internet of things (IoT) paradigm has been utilized in various healthcare domains for analyzing the realtime data and recommendations upon it. IoT is playing an important role in those applications specifically which involve ubiquitous sensors and actuators communicating through wireless sensor network (WSN) towards solutions of many problems. Most of the applications today highly demand for faster processing of generated data [1]. Furthermore, many issues arise such as data volume, velocity, and variation due to the utilization of sensors, mobility, and geographic distribution in addition to the requirements for accuracy, security, quality of service (QoS), and operational costs [2].

In recent years, cloud computing technology has been widely adopted in IoT-enabled healthcare applications to provide scalability, data analysis, and reliability [3]. The geographic distribution of cloud data centers where processing of data collected from sensors requires transmission through multihop distances affects the delay-sensitive healthcare applications. Moreover, healthcare applications environments are heterogeneous in nature, so managing the cloud of resource allocations for uneven and uncertain data loads coming from healthcare solutions is a very complex task [4]. There are certain issues regarding cloud computing commonly reported in the literature as follows [4]:

(i) Sending huge amount of data to the virtual computing platform causes significant overhead in terms of time, throughput, energy consumption, and cost

(ii) The cloud may be physically located as very far away, so it cannot service IoT application with reasonable latency and throughput 
(iii) Data centers may be overloaded to process large amount of big data in real time and may lead to facing challenges, i.e., capacity, security, and analytics

(iv) Cloud computing is hard to accommodate analytic engines for efficient processing of big data

Fog computing is one of the promising solutions for healthcare applications to explore lightweight and computing resources close to the IoT data source [5]. Fog nodes are equipped with computational infrastructure, services, and management models to execute data processing closer to the edge of network and provide opportunities in the form of reducing latency, reducing the need for multihop data communication, distributing resource demands, and promoting service flexibility. Healthcare data are delay sensitive which need to be process in real time to make timely decisions on critical patient's health. In one way, fog computing helps in reducing delays but in the other way, fog nodes consume power and energy because most of the time, these nodes remain active to process healthcare data. In order to overcome this problem, this study proposed a framework with a clustering method helping to minimize the energy consumption.

Big data is considered as a large amount of structure, unstructured, and semistructured data which are continuously generated and received by the hospitals [6]. According to the current organization understandings, one way to deal with the big data is to apply analytics to their big data and get useful insights out of it [7]. Based on the literature review, we discussed and suggested important services of big data infrastructure which need to be present in fog devices for analysis of healthcare data.

The rest of the paper is organized as follows: the state of the art is discussed in Section 2. An overview of fog computing platform is given in Section 3. A framework for healthcare solution is proposed and a hospital case scenario is explained in Section 4. Experimental setup, parameters, and results are discussed in Section 5. The big data analytics and its infrastructure containing important components are discussed in detail in Section 6. Finally, the paper is concluded in Section 7.

\section{Related Work}

This section describes the state of the art in order to understand the relevant studies carried out in the literature. The authors selected the most relevant articles for the literature review which correspond to the healthcare applications. These are discussed as follows.

Gia et al. [8] proposed a health monitoring system based on fog computing, and this system includes facilities, i.e., data mining, storing, and notification at the edge of architecture. In this study, the authors explored how the ECG extraction is arranged. Template-based technique known as feature extraction is used to analyse the ECG signals. Bandwidth usage and service delivery are found to be efficient in the experimental results.
Doukas and Maglogiannis [9] presented the online data management where processing of IoT-enabled pervasive applications is handled by the cloud. The proposed prototype is able to receive patient data from the IoT devices and finally process them in the cloud. Issues related to security are observed among the entities during communication. Important features of the prototype, i.e., Representational State Transfer (REST) API-based acce,s; scalability, and interapplication interoperability, are considered.

According to the Renta et al. [10], healthcare data received from the IoT distributed devices are focused to be stored in remote cloud. Data management system consists of IoT devices which collect the patient data in real time. This study revealed that data stored in cloud processed quickly and subscribed users can get quick notification during emergency. The alert system is also presented based on the predefined health rules and users' reactions.

Chen et al. [11] considered the security aspects of medical data shared through cloudlet data collected through encryption. A trust model is proposed to identify reliable entities to share the data i.e. hospital, doctors, chambers etc. Moreover, the trust model also used to connect medical professionals and patients. During data sharing, data are segmented into three parts and stored in the cloud. The intrusion detection system (IDS) remains active throughout the whole process to prevent malicious attacks.

Mahmud et al. [12] proposed a framework to perform data analysis and visualization in order to predict health shocks based on predefined data set. This framework depends on cloud platform and inclusion of Amazon web services (AWS), geographical information system (GIS), and fuzzy rule-based summarized techniques. The framework provides the opportunity to classify health shocks with accuracy using a data model. Moreover, it can explain the casual factors of health with the help of linguistic rules.

Zhang et al. [13] introduced Health-CPS, a cyberphysical system, aimed at providing convenient and efficient healthcare service to patients. The system depends on the cloud computing and data analytics to solve various big data-related issues of healthcare applications. The proposed system consists of layers, i.e., data collection layer, data management layer, and data-oriented service layer. The collection of data is performed in the unified standard which supports distributed storage and parallel processing.

Fazio et al. [14] designed an e-health Remote Patient Monitoring (RPM) system using cloud platform called FIWARE. Their main focus is to speed up the development of RPM availing the facilities from FIWARE. Patients are assisted to optimize the responsibilities of medical professionals. The implementation of FIWARE cloud to the RPM enhanced modularity, scalability, and efficiency.

Peddi et al. [15] proposed a e-health calorie system based on the cloud. The system can classify accurately different food objects from the meal and compute the overall calories. This system is able to do computation offloading from mobile e-health applications to the cloud. Cloud platform provides accurate outcome with tolerable latency, after the resources are managed by broker entity in the cloud. The 
broker is used to manage dynamic cloud allocation mechanism in real time if there is demand.

Jindal [16] proposed a technique to calculate heart rate with the help of embedded sensors and photoplethysmography signals in smart phone. This proposal consists of three steps of data processing. This technique is required to be associated with cloud to select accurate PPG signals through deep learning mechanisms and classify the signals into estimated heart rates. The TROIKA dataset is used for the evaluation of this technique. They concluded that this technique brought accurate heart rate predictions.

Muhammad et al. [17] explained healthcare solution for voice pathology monitoring users. As a cloud-based platform, this proposed solution used a voice pathology detection system that incorporates local binary pattern on voice signal produced through MelSpectrum technique. Pathodology conduction is done by the machine learning classifier. Results showed that with the merging of cloud platform, the accuracy and accessibility of the healthcare solutions improved.

Gupta et al. [18] discussed a cloud-based IoT-enabled predictive physical activity analysis model. Embedded sensors, cloud computing, and XML web services are used in this model for the faster, secure, and efficient data collection, processing, and communication. Different perspectives were taken, i.e., service adaptation, prediction analysis, and efficiency for the evaluation of this model. The proposed model can send an alert to the responsible person to notify the abnormality.

Real-time health issues of aged and disable people were discussed by Hossain and Muhammad [19]. The proposed Health-IoT can monitor track and store healthcare data for treatment. The Health-IoT framework is able to collect ECG data from smart phones and sensors. The data can be transferred to the cloud where doctors can access and assess it. Data analytics are applied on the data to find out any errors in data and to detect abnormality.

According to the work of Gia et al. [20], the remote monitoring of cardiac patients can be made possible at low cost through their fog-based health monitoring system. The system includes energy-efficient IoT sensors and smart gateways. ECG, body temperature, and respiration rate data are collected by the sensors and are sent to the gateways though wireless for autoanalysis and notifications. Furthermore, it can also help in visualizing the outcome in an efficient way.

Fog-based healthcare framework is proposed by Ahmad et al. [21]. The framework is considered as the middle layer between cloud platform and end IoT devices. Cloud access security broker (CASB) is used with the framework to enhance data privacy and security of healthcare data. The framework is able to aggregate data from several sources with decent cryptographic assessment.

Chakraborty et al. [22] proposed a fog-based computation platform where latency-sensitive health data are considered. In their proposed programming model, geographically distributed large-scale healthcare application is handled. Evaluation of this model is done through processing heart rate healthcare data. The fog-based healthcare solution improved data accuracy, service delivery, and data consistency.

Service-oriented architecture of fog computing is discussed by Dubey and Constant [23] where validation and evaluation of raw health data are sensed through IoT devices. The proposed system with resource-constrained embedded computing instances is able to conduct the data mining and data analysis. Furthermore, these instances are also capable of identifying important patterns from the health data and forwarding them to the cloud for further storage and usage. The main theme of this study is to highlight the big data processing with low-power fog resources.

Negash et al. [5] implemented a smart e-health gateway of fog computing for IoT-enabled health-care services. All the smart gateways that were distributed geographically were used to manage IoT devices connected with the patients. Clusters of gateways were formed to perform data analytics and configurations. The proposed system was responsible for monitoring patient's movement independently.

Rahmani et al. [24] proposed an e-health gateway system where the smart gateways are placed in correct places to offer real-time storage, processing, and analytics. The system overcomes the issues related to the mobility, energy, and reliability. The authors also developed a prototype called UTGATE which is based on the concept of smart e-health gateway. System performance is evaluated through the IoTbased early warning score (EWS).

\section{Fog Computing Platform}

This section aims to give an overview of fog computing and its components. Furthermore, fog computing is shown with the help of diagram to help readers better understand it.

The environment of fog computing consists of fog nodes which perform diverse computational tasks at the edge of the network as illustrated in Figure 1. There can be many fog levels that are arranged in a network to form in hierarchically distributed way. Each fog node is equipped with memory, storage, network bandwidth, and processing cores. In a particular hospital scenario, the sensors deployed in hospital collect the data and forward to the fog nodes for further processing. In fog nodes, the resources such as memory, storage, cores, bandwidth, etc. are virtual and can be shared through MCI (Micro Computing Instances) [25].

As we assume, all the fog nodes in healthcare solution are always active to perform computation of delay-sensitive healthcare data, so an energy-efficient network needs to be designed to minimize the energy consumption of the whole network. Therefore, we aim to propose an energy-efficient fog-based solution for healthcare.

\section{IoT Healthcare Solution and Proposed Framework}

In this section, a fog-based energy-efficient wireless sensor network healthcare solution is proposed. Based on the literature review, a healthcare solution framework is discussed. We aim to focus on energy-efficient wireless sensor network fog-based architecture where the energy usage of sensors is 


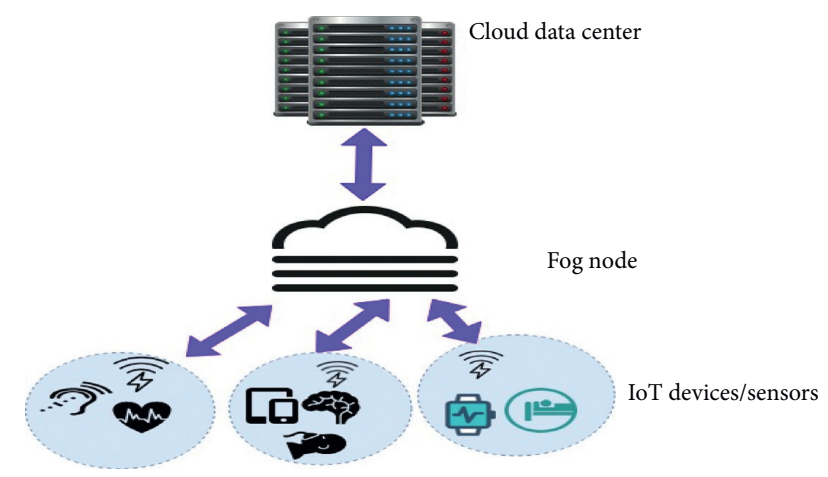

Figure 1: General fog computing environment.

to be minimized in order to prolong the network lifetime of sensor nodes at bottom layer. Furthermore, big data collected by these sensors are to be analyzed at the fog nodes. Literature review leads us to provide the answer to the following key research questions:

(i) RQ1: How to increase the lifetime of the sensor network use in the IoT healthcare applications?

(ii) RQ2: What are the important services that need to be present in fog nodes to provide analytics?

As Figure 2 depicts, the fog computing environment consists of special networking devices called fog nodes which perform various computational tasks at the edge of network. Every fog node has the capability of providing services, i.e., processing, memory, and storage and network bandwidth. We place these devices in the middle layer of our proposed architecture.

In the bottom layer, wearable sensors are deployed on the body of the patients and used to collect a vast amount of data. These wearable sensors monitor and collect patient's physiological data in the form of ECG, blood oxygen, and other health-related information. Deployed sensors help the patients to reduce their inconvenience of regular visits to the doctor [26].

Wearable sensors use in the bottom layer could have limited power, memory, processing, and communication, so we aim to implement a clustering method which is used to maximize the network lifetime of wireless sensor network [27].

The middle layer is composed of fog nodes which are composed of processing, storing, memory, and network bandwidth capabilities. As the healthcare data collected by the wearable devices at the bottom layer can be increased in size, there is a need to carry out data mining and analytics on such big data. Fog node at the middle layer can process the raw data collected from the bottom layer and carries out analytics [28].

The important healthcare data analyzed in the fog node (middle layer) are processed immediately; otherwise, top layer consisting of cloud computing is responsible for further storage and processing [27].

Regarding the proposed framework of this study, it is necessary to design energy-efficient sensor network in the bottom layer where energy usage of healthcare sensor nodes

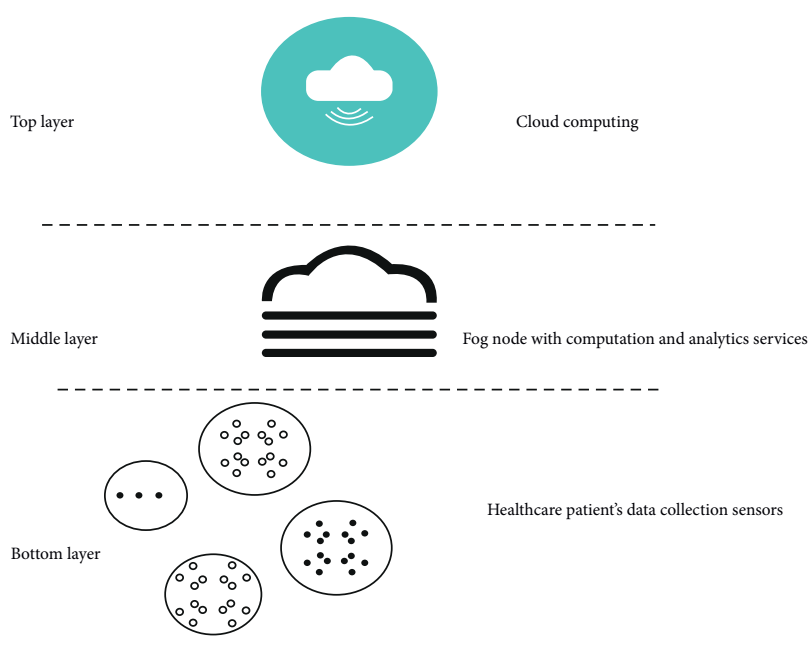

FIGURE 2: The three layers in the proposed framework.

can be minimized before it is transmitted to the middle layer such as fog node for further analytics. Therefore, a hospital scenario has been discussed with the clustering technique to minimize the energy usage of sensor nodes inside the hospital.

4.1. Case Study: Hospital Scenario. This section aims to address the RQ1 as aforementioned. The authors discussed a hospital scenario as a case study which help readers to understand the useful implementation of fog nodes inside the hospital and how clustering method could help in energy-efficient network.

Clustering is a technique used to increase the network lifetime and energy efficiency. Sensor nodes can be organized into groups called clusters. Each cluster contains cluster members and cluster head $(\mathrm{CH})$, where cluster members send the data packets to the cluster head, and cluster head aggregates and gathers the data and finally forwards the data to the base station. Comparing both types of sensor nodes in terms of energy usage, cluster heads consume more energy.

In WSN, sensor nodes are small in size, having low power, communication, and computing properties. WSN (Wireless Sensor Network) contains thousands of nodes which can be spatially distributed in various locations to monitor physical, environmental, medical, etc. conditions. Lifetime of sensor nodes depends on the batteries inside these sensors, and it is impractical to change the batteries of every sensor node due to the huge network scale. Therefore, it is important to consider the efficient energy usage of these networks before designing any topology [27].

Regarding the proposed framework, it is necessary to design energy-efficient sensor network in the bottom layer where energy usage of healthcare sensor nodes can be minimized before it is transmitted to the middle layer, i.e., fog node, for analytics.

Figure 3 illustrates various kinds of sensors and IoT devices used inside the hospital in our proposed framework. These sensors collect patient's health data, and the collected 


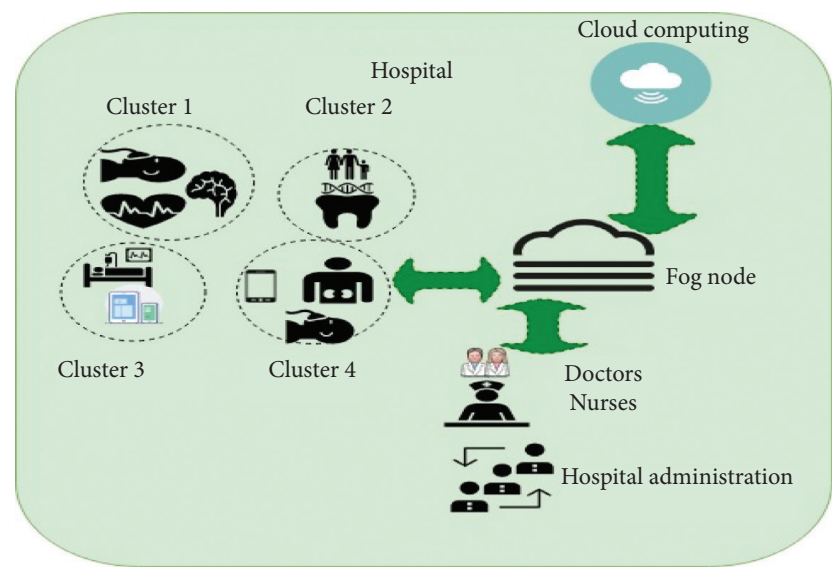

FIgURE 3: Hospital scenario where the clustering method is performed.

data are sent to the fog node for analytics. The data needed on immediate basis are processed by the fog node and can be easily accessed by the doctors, health professionals, and administration staff in the hospital for decisions. The data not needed immediately is sent to the cloud for future use.

It must be noted that all the IoT devices and sensors are battery-powered and should be utilized in an efficient way. Furthermore, some of the IoT devices have built-in batteries so it is not an easy to task to recharge or replace the batteries every time, especially in hospital scenario. Also, it is to be noted that IoT devices deployed in hospital send data continuously; in this way, energy utilization increases with the number of transmissions too. To solve this issue, we have proposed a framework based on clustering technique, which will group the number of IoT devices into clusters. The IoT device with high energy and processing capability will be cluster head $(\mathrm{CH})$. Other IoT devices near the $\mathrm{CH}$ will become members in respective clusters. When a $\mathrm{CH}$ gathers data from other cluster heads, it performs aggregation and forwards to the fog node. The IoT nodes in every floor of the hospital can organize as a cluster.

We know that healthcare data are delay sensitive. These data need to be processed on time to be analyzed. To solve this problem, fog node is placed in the edge of the network to process the data immediately. The healthcare data processed by the fog node can be further accessed by the doctors, healthcare professionals, and administration staff inside the hospital. Furthermore, certain issues arise by using the cloud computing discussed as follows:

(i) Sending huge amount of data to the virtual computing platform causes significant overhead in terms of time, throughput, energy consumption, and cost

(ii) The cloud may be physically located as very far away, so it cannot service IoT application with reasonable latency and throughput

(iii) Data centers may be overloaded to process large amount of big data in real time and may lead to facing challenges, i.e., capacity, security, and analytics (iv) Cloud computing is hard to accommodate analytic engines for efficient processing of big data

To overcome these challenges, data analytics is performed at the edge of the network called fog. This fog node can be placed near where the data are generated [29-32].

\section{Experimental Setup}

This section discusses the details about the parameters used for experiments. Furthermore, the authors briefly explain the experimental setup and the results they achieved after the simulations.

A proposed IoT healthcare fog-based solution is compared with cloud-based solution using IFogSim [33]. Although there are various simulators used today for simulation of fog computing, due to the high availability of its source code on GitHub, a lot of target audience, and its easy graphical user interface (GUI), we decided to use IFogSim in our simulations. IFogSim has the ability to integrate various resource management techniques which can be further customized depending on the research area. It is high-performance simulator and its association with CloudSim makes it more useful. CloudSim is very efficient tool in simulation of cloud-based environments [33]. The performance of the proposed solution is compared to the cloud-based solution in terms of network delay and energy usage. Simulation parameters are given in Table 1.

Both healthcare solutions are compared and investigated. After careful investigation, our proposed fog-based big data healthcare solution performs well. In the following, we aim to discuss the measured parameters used in the simulations.

5.1. Network Delay. In the experiment, it is observed that average network delay becomes high in cloud healthcare solution because the same communication link shared in cloud by multiple healthcare applications reduces the bandwidth. Furthermore, we observed an increase in the network congestion and a higher round trip time. In contrast, network delay found in fog-based healthcare solution was low as there were multiple communication links present between data source and proximate computing components. Also, the cluster head node is responsible for controlling the data flow to reduce the network delay as mentioned in Figure 4 .

5.2. Energy Usage. Single virtual machines (VM) in cloudbased healthcare solution execute applications where in fogbased healthcare solution multiple MCIs (micro computing instances) execute an application collectively. MCI used in fog-based healthcare solution consumes fewer amounts of energy and is lightweight as compared to the VM of cloudbased healthcare solution. It is observed from the experiment that the overall energy usage of MCIs was less than the VMs, even in the case of increasing the number of applications load on MCIs as shown in Figure 5. 
TABLE 1: Simulation parameters.

\begin{tabular}{lc}
\hline Parameter & Value \\
\hline Simulation duration & $400 \mathrm{sec}$ \\
Cloud data center & \\
$\quad$ Network latency & $10 \mathrm{~ms}$ \\
$\quad$ Energy consumption of VMs & $10-15$ megaJoules \\
$\quad$ Average VMs per server & $10-15$ \\
Fog cluster & $10 \mathrm{~ms}$ \\
$\quad$ Network latency & $2-3$ megaJoules \\
Energy consumption of MCIs & $3-10$ \\
$\quad$ Average MCIs per server & $25 \times 25 \mathrm{~m}$ \\
Network size (fog- and cloud-based solution) & \\
\hline
\end{tabular}

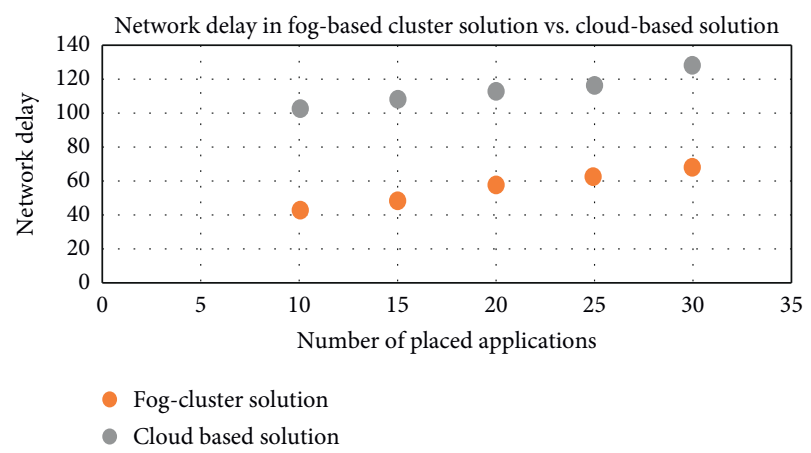

FIgURE 4: Network delay in fog-based and cloud-based solution.

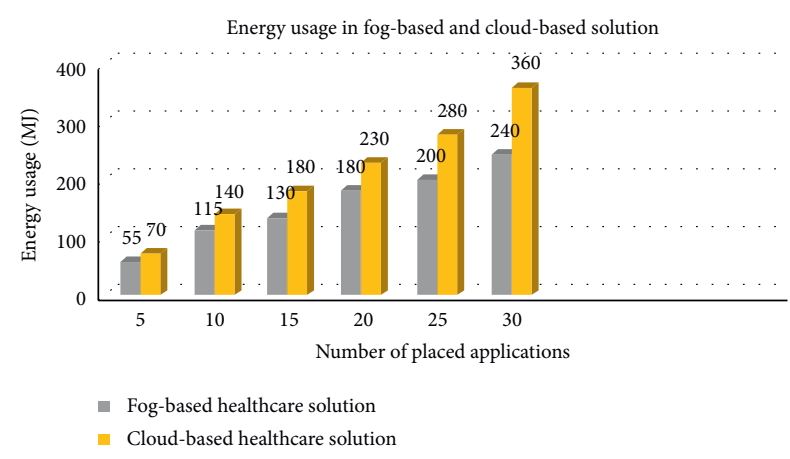

Figure 5: Energy usage in fog- and cloud-based solutions.

\section{Big Data Analytics}

The authors aim to give a brief discussion about big data, its infrastructure with important components which must be present to better analyse the big data. Moreover, how to use data analytics in fog devices is discussed. In the end of the section, our hospital scenario is discussed in the context of big data analytics in fog devices.

Large organizations, companies, and research centers receive terabytes of data from various sources, i.e., social media, e-mails from customers, survey responses, phone calls, web server logs, and IoT nodes. Big data is considered as large amount of structure, unstructured and semistructured data, which are continuously generated and received by the organizations [34]. According to the current organization understanding, one way to deal with the big data is to apply analytics to their big data and get useful insights out of it. Big data is a kind of advanced analytics where complex applications such as predictive models and statistical algorithms are involved. Big data help to examine a large amount data and extract/uncover hidden patterns from it. Big data can be analyzed in batch mode and streamline mode. It means that for some applications, data are analyzed and results are generated on store-and-process paradigm basis [7].

6.1. Big Data Analytics Infrastructure. In this section, we aim to answer the research questions 2 as mentioned above. According to Tang et al. [35], a typical big data infrastructure consists of the following components and layers. The big data platforms have the capabilities of integrating, managing, and applying efficient computational processing to the data. Furthermore, these platforms are used to optimize complex manipulations of large amount of data and considered as big data execution engine. Handling big data with traditional databases is impossible due to its performance/ cost towards processing:

(i) Data management helps any organization to produce data in high-quality format for efficient analysis. Steps included in data management are cleaning, removing anomalies, and transformations to the desired format. Once the steps are performed, the organization must create master data management program for the objective of best analysis.

(ii) Storage is another important component where a large amount of data is stored because traditional warehouses are not good with storing unstructured and semistructured data.

(iii) Analytics core functions include data mining that help to examine a large amount of data and discover patterns from it. This information can be further analyzed to help answer complex questions.

(iv) Presentation is the displaying of information in intuitive and graphical form that help organizations to extract insights for decision making.

6.2. Data Analytics in the Fog Devices. In the context of cloud computing, data generated by the IoT devices and sensors are collected and transferred to the cloud for further processing and storage. Although it works well, it poses some challenges; for example, applications require real-time processing, shortening communication time and its cost. Fog computing helps to process the data before they are transferred to the cloud and provides many benefits in the form of shortening communication time and cost and minimizing the need of huge data storage. In short, it is the best solution for all IoT applications [36].

Fog devices are capable of providing low latency and context awareness while cloud providing globalization, so some applications achieve their goals using fog computing 


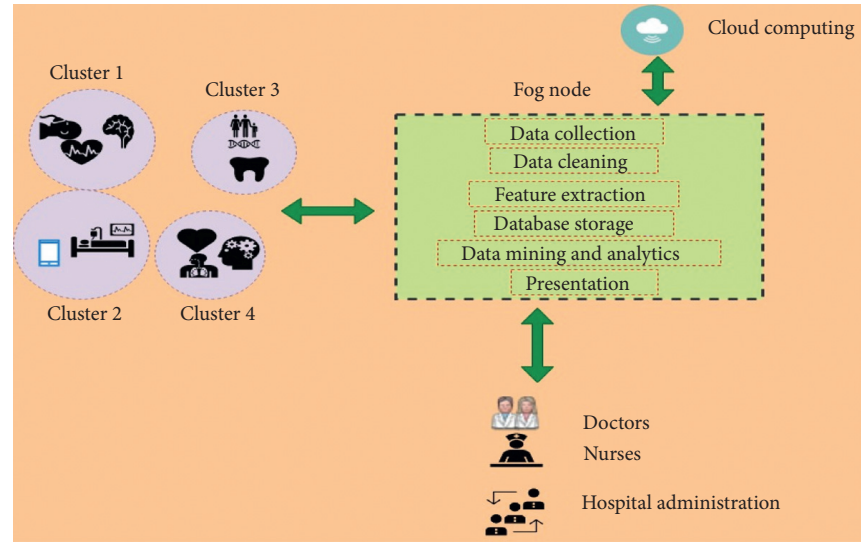

Figure 6: Data analytics in fog node.

and cloud computing in the form of localization and globalization [36]. All the fog devices need to have data interfaces, integration with the cloud, handling of incoming continuous data with streaming analytics, and reliable network architecture for moving the real-time processing functions to the edge. Less-time sensitive data can be moved to the cloud for long-term storage and historical analysis. Machine learning and visualization functions need to be applied for improving the performance of IoT applications [6].

6.3. Big Data Analytics. Based on the literature review regarding data analytics in fog nodes, we aim to discuss hospital scenario in the context of big data analytics.

As illustrated in Figure 6, according to the literature, the authors suggest six important features that must be present in the fog nodes used for data analytics. Data are collected by the fog nodes coming from various sensors deployed in hospital. Cleaning the data process involves the identification of inaccurate or errors in data and removing them to avoid full storage. Feature extraction is the ability to reduce the raw data into more managed groups for processing. Also, it helps to reduce the number of redundant hospital data for analysis by the doctors, nurses, and administration staff. In the above scenario, data mining is applied with the help of machine learning algorithm to extract and find data from disparate systems. In addition, it supports providing services in healthcare system such as identifying unnecessary utilization of high-cost services, e.g., imaging tests and emergency department, understanding the flow of patients through the hospital, identifying the patients diagnosed for diabetes, etc. [37]. Finally, the data are presented in graph or text format which help the doctors, nurses, or other hospital's management staff in making useful decisions to diagnose the patients [37].

\section{Conclusions}

In this study, the performance of the proposed solution is compared to the cloud-based solution in terms of network delay and energy usage. The parameters used in the experiment were network delay and energy usage. The average network delay becomes high in cloud-based healthcare solution because the same communication link shared in cloud by multiple healthcare applications reduces the bandwidth. Furthermore, we observed an increase in the network congestion and a higher round trip time. In contrast, network delay found in fog-based healthcare solution was low as there were multiple communication links present between data source and proximate computing components.

Regarding energy usage parameter, we observed that the overall energy usage of MCIs was less than the VMs even in the case of increasing the number of applications load on MCIs. An overview of big data analytics and its infrastructure is discussed. According to the literature review, fog computing supports many applications. Here, we need to describe how our energy-efficient framework can be supported by other domains. The first typical example is smart home where many devices inside the home are connected which require high computing power; if our proposed framework is properly implemented inside the smart home, it would be more helpful in minimizing the energy cost of the network. Another domain to implement the proposed framework in vehicles is called vehicular ad hoc network in which fog nodes are responsible for receiving/sending data from vehicles or other fog nodes, to help in prolonging the lifetime of network through clustering method. In the future, we plan to simulate other parameters in experiments such as instances cost of cloudand fog-based solutions, to investigate CPU utilization after varying the number of sensors. This study was focused on proposing energy-efficient framework; in the future, we would like to add an important functionality such as secure connection between end devices in order to bring a desired performance of fog computing. We also have a plan to do simulation using another simulator which supports fog computing and compare the results from both simulators. And last but not the least, mathematical modeling of the proposed framework will be introduced.

\section{Data Availability}

The data used to support the findings of this study are included within the article. 


\section{Conflicts of Interest}

The authors declare that they have no conflicts of interest regarding the publication of this paper.

\section{Acknowledgments}

This work was supported by the National Key R\&D Program of China (No. 2017YFB1002101).

\section{References}

[1] S. M. R. Islam, D. Kwak, M. H. Kabir, M. Hossain, and K.-S. Kwak, "The internet of things for health care: a comprehensive survey," IEEE Access, vol. 3, pp. 678-708, 2015.

[2] F. Fernandez and G. C. Pallis, "Opportunities and challenges of the internet of things for health-care: systems engineering perspective," in Proceedings of the 4th International Conference on Wireless Mobile Communication and Health-CareTransforming Health-Care through Innovations in Mobile and Wireless Technologies (MOBIHEALTH), IEEE, Athens, Greece, 2014.

[3] M. Hassanalieragh, A. Page, T. Soyata et al., "Health monitoring and management using internet-of-things (IoT) sensing with cloud-based processing: opportunities and challenges," in Proceedings of the IEEE International Conference on Services Computing, IEEE, New York, NY, USA, July 2015.

[4] F. Bonomi, R. Milito, J. Zhu, and S. Addepalli, "Fog computing and its role in the internet of things," in Proceedings of the First Edition of the MCC Workshop on Mobile Cloud Computing, Helsinki, Finland, August 2012.

[5] B. Negash, T. N. Gia, and A. Anzanpour, "Leveraging fog computing for health-care iot," Fog Computing in the Internet of Things, pp. 145-169, Springer, Berlin, Germany, 2018.

[6] H. Dubey, "Fog data: enhancing telehealth big data through fog computing," in Proceedings of the ASE Bigdata \& Socialinformatics, pp. 1-6, Kaohsiung Taiwan, October 2015.

[7] B. Javadi, B. Zhang, and M. Taufer, "Bandwidth modeling in large distributed systems for big data applications," in Proceedings of the 15th International Conference on Parallel and Distributed Computing, Applications and Technologies, IEEE, Hong Kong, China, December 2014.

[8] T. N. Gia, M. Jiang, A. Rahmani, T. Westerlund, P. Liljeberg, and $\mathrm{H}$. Tenhunen, "Fog computing in healthcare internet of things: a case study on ECG feature extraction," in Proceedings of the 2015 IEEE International Conference on Computer and Information Technology; Ubiquitous Computing and Communications; Dependable, pp. 356-363, Autonomic and Secure Computing; Pervasive Intelligence and Computing, Liverpool, UK, 2015.

[9] C. Doukas and I. Maglogiannis, "Bringing IoT and cloud computing towards pervasive health-care," in Proceedings of the Sixth International Conference on Innovative Mobile and Internet Services in Ubiquitous Computing, pp. 922-926, IEEE, Palermo, Italy, July 2012.

[10] T. R. Renta, S. Sotiriadis, and E. G. M. Petrakis, "Health-care sensor data management on the cloud," in Proceedings of the 2017 Workshop on Adaptive Resource Management and Scheduling for Cloud Computing (ARMS-CC'17), pp. 25-30, ACM, New York, NY, USA, 2017.

[11] M. Chen, Y. Qian, J. Chen, K. Hwang, S. Mao, and L. Hu, "Privacy protection and intrusion avoidance for cloudlet- based medical data sharing," IEEE Transactions on Cloud Computing, 2016.

[12] S. Mahmud, R. Iqbal, and F. Doctor, "Cloud enabled data analytics and visualization framework for health-shocks prediction," Future Generation Computer Systems, vol. 65, pp. 169-181, 2016.

[13] Y. Zhang, M. Qiu, C. Tsai, M. M. Hassan, and A. Alamri, "Health-CPS: health-care cyber-physical system assisted by cloud and big data," IEEE Systems Journal, vol. 11, no. 1, pp. 88-95, 2015.

[14] M. Fazio, A. Celesti, F. G. Marquez, A. Glikson, and M. Villari, "Exploiting the FIWARE cloud platform to develop a remote patient monitoring system," in Proceedings of the 2015 IEEE Symposium on Computers and Communication (ISCC), IEEE, Larnaca, Cyprus, 2015.

[15] S. V. B. Peddi, P. Kuhad, A. Yassine, P. Pouladzadeh, S. Shirmohammadi, and A. A. N. Shirehjini, "An intelligent cloud-based data processing broker for mobile e-health multimedia applications," Future Generation Computer Systems, vol. 66, pp. 71-86, 2017.

[16] V. Jindal, "Integrating mobile and cloud for PPG signal selection to monitor heart rate during intensive physical exercise," in Proceedings of the 2016 IEEE/ACM International Conference on Mobile Software Engineering and Systems (MOBILESoft), IEEE, Austin, TX, USA, May 2016.

[17] G. Muhammad, S. M. M. Rahman, A. Alelaiwi, and A. Alamri, "Smart health solution integrating IoT and cloud: a case study of voice pathology monitoring," IEEE Communications Magazine, vol. 55, no. 1, pp. 69-73, 2017.

[18] P. K. Gupta, B. T. Maharaj, and R. Malekian, "A novel and secure IoT based cloud centric architecture to perform predictive analysis of users activities in sustainable health centres," Multimedia Tools and Applications, vol. 76, no. 18, pp. 18489-18512, 2017.

[19] M. S. Hossain and G. Muhammad, "Cloud-assisted industrial internet of things (IIoT)-enabled framework for health monitoring," Computer Networks, vol. 101, pp. 192-202, 2016.

[20] T. N. Gia, M. Jiang, V. K. Sarker et al., "Low-cost fog-assisted health-care IoT system with energy-efficient sensor nodes," in Proceedings of the 2017 13th International Wireless Communications and Mobile Computing Conference (IWCMC), IEEE, Valencia, Spain, June 2017.

[21] M. Ahmad, M. B. Amin, S. Hussain, B. H. Kang, T. Cheong, and S. Lee, "Health fog: a novel framework for health and wellness applications," The Journal of Supercomputing, vol. 72, no. 10, pp. 3677-3695, 2016.

[22] S. Chakraborty, S. Bhowmick, P. Talaga, and D. P. Agrawal, "Fog networks in health-care application," in Proceedings of the 2016 IEEE 13th International Conference on Mobile Ad Hoc and Sensor Systems (MASS), IEEE, Brasilia, Brazil, October 2016.

[23] H. Dubey and N. P. Constant, "Fog data: enhancing telehealth big data through fog computing," Proceedings of the ASE Bigdata \& Socialinformatics, vol. 14, pp. 1-6, 2015.

[24] A. M. Rahmani, T. N. Gia, B. Negash et al., "Exploiting smart e-Health gateways at the edge of healthcare Internet-ofThings: A fog computing approach," Future Generation Computer Systems, vol. 78, pp. 641-658, 2018.

[25] R. Mahmud, S. N. Srirama, K. Ramamohanarao, and R. Buyya, "Quality of experience (QoE)-aware placement of applications in fog computing environments," Journal of Parallel and Distributed Computing, vol. 132, pp. 190-203, 2019. 
[26] S. C. Mukhopadhyay, "Wearable sensors for human activity monitoring: a review," IEEE Sensors Journal, vol. 15, no. 3, pp. 1321-1330, 2014.

[27] J. Wang, Y. Gao, W. Liu, A. Sangaiah, and H.-J. Kim, “An improved routing schema with special clustering using PSO algorithm for heterogeneous wireless sensor network," Sensors, vol. 19, no. 3, p. 671, 2019.

[28] H. Cao and M. Wachowicz, "An edge-fog-cloud architecture of streaming analytics for internet of things applications," Sensors, vol. 19, no. 16, p. 3594, 2019.

[29] M. Satyanarayanan, P. Simoens, Y. Xiao et al., "Edge analytics in the internet of things," IEEE Pervasive Computing, vol. 14, no. 2, pp. 24-31, 2015.

[30] W. Shi, J. Cao, Q. Zhang, Y. Li, and L. Xu, "Edge computing: vision and challenges," IEEE Internet of Things Journal, vol. 3, no. 5, pp. 637-646, 2016.

[31] L. M. Vaquero and L. Rodero-Merino, "Finding your way in the fog," ACM SIGCOMM Computer Communication Review, vol. 44, no. 5, pp. 27-32, 2014.

[32] S. Yi, L. Cheng, and Q. Li, "A survey of fog computing: concepts, applications and issues," in Proceedings of the 2015 Workshop on Mobile Big Data, Hangzhou, China, June 2015.

[33] H. Gupta, A. Vahid Dastjerdi, S. K. Ghosh, and R. Buyya, "iFogsim: a toolkit for modeling and simulation of resource management techniques in the internet of things, edge and fog computing environments," Software: Practice and Experience, vol. 47, no. 9, pp. 1275-1296, 2017.

[34] F. Mehdipour, N. Hamid, and B. Javadi, "Energy-efficient big data analytics in datacenters," Advances in Computers, vol. 100, pp. 59-101, 2016.

[35] B. Tang, Z. Chen, T. Wao et al., "A hierarchical distributed fog computing architecture for big data analysis in smart cities," in Proceedings of the ASE Bigdata \& SocialInformatics, Kaohsiung Taiwan, October 2015.

[36] F. Bonomi, "Fog computing and its role in the internet of things," in Proceedings of the First Edition of the MCC Workshop on Mobile Cloud Computing, Helsinki, Finland, August 2012.

[37] HealthIT Analytics, "Data mining, big data analytics in health-care: what's the difference?," 2020, https:// healthitanalytics.com/news/data-mining-big-data-analyticsin-health-care-whats-the-difference.

[38] Sensors Facilitate Health Monitoring, 2019, https://www. fierceelectronics.com/components/sensors-facilitate-healthmonitoring. 